resorted to only in very bad cases, in which a high mortality will be the rule no matter what treatment is adopted.

We must base our criticism on the extent of injury done to the cervix. Looking at the matter from that point of view, I find that in six cases in which the os was the size of a shilling or less I had one bad case of laceration and two of very slight laceration. In fourteen cases recently reported by Gardner from the practice of the Maternity Hospital, two had very bad lacerations, one being the case described already. Leopold, one of the strongest advocates of this treatment, has reported seventeen cases without a laceration of any account, although in Case II of his first series there was a laceration, for he had to apply stitches on account of the bleeding. Leopold's results are very satisfactory, but if you read over the cases you will find that only in one was the cervix not already dilated to some extent. In his second paper he says: "The os was in some 1 pfennig, in others 1,3 , or 5 markpiece in size."

Now, there are certain factors which influence the occurrence of lacerations, and of these $I$ would mention the following:

1. The manner in which the dilatation is carried out.

2. The number of the pregnancy.

3. The degree to which the cervix has been taken up.

4. The age of the pregnancy.

The influence of the first two factors is obvious. It is perfectly clear that lacerations will be more common when dilatation is carried out rapidly than when it is done slowly, and in primiparae than in multiparae. There remain therefore only the other two to discuss, and they are really the most important. With comparatively few exceptions in the cases which have been reported by different writers, the cervix has been already taken up. Simpson and others, in this country, Germany, and America have, however, recorded cases where the cervix was not obliterated, and the os was not dilated. With time and caution doubtless these cases, if at or near term, can be safely dilated with Bossi or Frommer's instrument, as the recorded cases illustrate. Some of the worst tears, however, have been in such cases. But although the unobliterated cervix at or near term dilates wonderfully well, any one who has dilated to the full extent such a cervix and then tried to deliver with forceps or by version will bear me out when I say that the os externum invariably contracts round the head. One is always disappointed; the canal is never so well dilated as one expects.

I turn now to the last factor, the age of the pregnancy. In dilating the cervix in gynaecological work I have more than once found the cervix give way not externally but internally. 1 can also remember once having seen the same thing in dilating the cervix in a case of abortion. That this may occur has been pointed out by several writers. It is further borne out by Case XII (abortion at fourth month), which Gardner describes as follows: "Two days after delivery haemorrhage occurred while an intrauterine douche was being given: examination showed that a clot had been washed from a pit or depression within the cervix."

And this brings me to say that the cervix tears readily in the early months of pregnancy, and consequently Bossi's dilator should be used with great caution in such cases. Also, and this is of the very greatest importance, the tear may not always be external; by that I mean that to the finger from the vagina the vaginal portion of the cervix may appear intact, yet if one carefully examines the cervical canal one will find a tear there. Lacerations are commonest in this group, and the case of extensive laceration described was an illustration of this.

Finally, I would sum up my views as regards Bossi's or Frommer's dilator as follows :

1. When the cervix is obliterated and the os will admit the dilator without difficulty, difatation can be carried out in about twenty-five minutes, and there will be little or no laceration.

2. In cases where the pregnancy has advanced to or near term, even although the cervix is not obliterated, dilatation may be accomplished with comparative safety to the cervix provided care, time, and patience are expended in the operation.

3. In cases of early pregnancy with the cervix unobliterated there is a decided risk of extensive lacerations, even although the greatest care is taken.

REFERENCE.

1 Fourteen Cases of Forcible Dilatation of the Os by Means of the Bossi Dilator, Journal of Obstetrics and Gynaecology of the British Empire, October, rgo3.

\section{OBSERVATIONS ON THE PATHOGENESIS OF GOUT.}

By CHALMERS WATSON, M.B., F.R.C.P.E., Physician, Eye, Ear, and Throat Department, Marshall Street Dispensary Edinburgh.

Prior to Garrod's great ãiscovery of the presence of uric acid in the blood in gout (1847), some authorities expressed the opinion that a derangement of the intestinal tract was the primary factor in the disease. This view was expressed by Van Swieten (1255 A.D.) in the following terms: "Indigestio viscorum pro origine proxima hujus morbi habetur." Since 1847 this theory has been abandoned, and in the writings of Garrod 1876, Duckworth 1889, Ewart 1896, Roberts 1897 , and Luff 1898, the possibility of gout being due to an intestinal auto-intoxication or infection is never considered. In 1901 the writer revived this old-time theory in the following terms: " $(a)$ There is ample evidence to prove that the uric acid in the blood is not the primary factor in gout, and (b) uric acid can be deposited in cartilages and other tissues even in considerable amount, without the association of any inflammatory phenomena. (I) The last-mentioned point clearly proves that uric acid is not the factor which causes the inflammatory phenomena characteristic of the acute attack; (2) What, then, are the toxic principles in the blood which possess the power of inducing the characteristic inflammation? and (3) what are the factors which determine the passage of these toxic substances from she blood into the tissues? In connexion with these queries we have to consider the all-important part played by the alimentary canal. Here we have doubtless one of the important keys to the solution of the problem."

Evidence for or against this theory is difficult to obtain from the human subject, and it is therefore advisable to have recourse to the study of the comparative pathology of the disease. Gout is a rare occurrence in the lower animals. The literature of the subject contains references to isolated records of the occurrence of uratic deposits in the tissues of lower animals, mainly wild animals in captivity, but detailed histological records are wanting. As an indication of the rarity of the disease in poultry, I may say that some years ago the writer obtained the assistance of the editors of leading poultry journals in his quest for "gouty fowls."

A considerable amount of pathological material was forthcoming; this comprised fowls which had died from tuberculosis, fowls which had been affected with swollen joints, others with large chronic inflammatory swellings of the feet, and fowls that had succumbed to disease unaccompanied by any lesions in the limbs, which werevisible post mortem. In no single instance was there any indication of uratic deposit in or about the joints, or in any of the tissues of the dead animals. Three years ago, however, I was fortunate enough to secure one typical case of gout in a fowl (cock). The animal was a year old. and was bred on the farm from which it was sent to me. The clinical record shows that the fowl was observed to be out of sorts for a few days, its comb became dusky, and it was found dead.

The present paper is a record of the post-mortem appearances of the case. Particular attention is directed to the histological appearances of the kidneys, pancreas, intestine, and tissues generally, the lesions described being regarded as characteristic of the invasion of the tissues by bacteria or their products. The facts recorded have a bearing on present theories regarding the disease, more especially the wellknown views of Ebstein, but the consideration of this point is beyond the scope of the present paper.

Necropsy.

r. The synovia in some joints was of the colour and consistence of cream; this readily gave the murexide reaction. When this creamy layer was washed off there was no visible lesion of the articular or other structures of the joints.

2. A deposit of sodium urate in some extra-articular tissues, mainly in relation to the smaller joints. This was specially abundant in relation to tendons, several of which were completely necrosed. Micnoscopic sections of these tendons, which were treated by the addition of weak hydrochloric acid, showed copious deposit of uric acid crystals, The murexide reaction was readily obtained.

3. The heart showed early pericarditis, a fine layer of fibrinous

1 Since the above was written my attention has been drawn to an admirable paper by Gore (BRITISH MEDICAL JOURNAL, I900, ii, p. 898), in which the same opinion is clearly expressed. 
exudation being present at the base of the organ. Microscopic examination showed pronounced distension: $c$ and a quantity of fibrin in the subepicardial veins.

4. Widespread thrombosis, notably in the vessels of the liver, lungs, kidneys, and some large abdominal veins. The distension and thrombosis of veins in the liver is shown in Fig. I. The appearances at the necropsy appeared to indicate that this thrombosis was the immediate necropsy appear

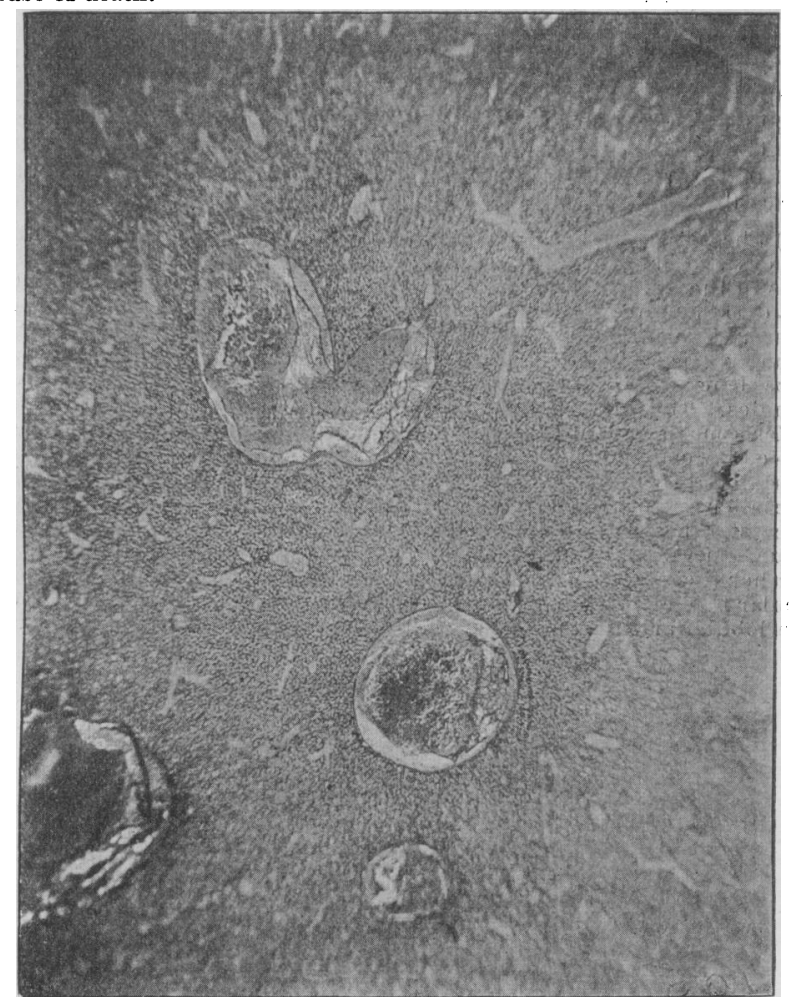

Fig. 1.-Gout in fowl. Liver. (Note the distel sion and thrombosis o the veins and the congestion of the organ.)

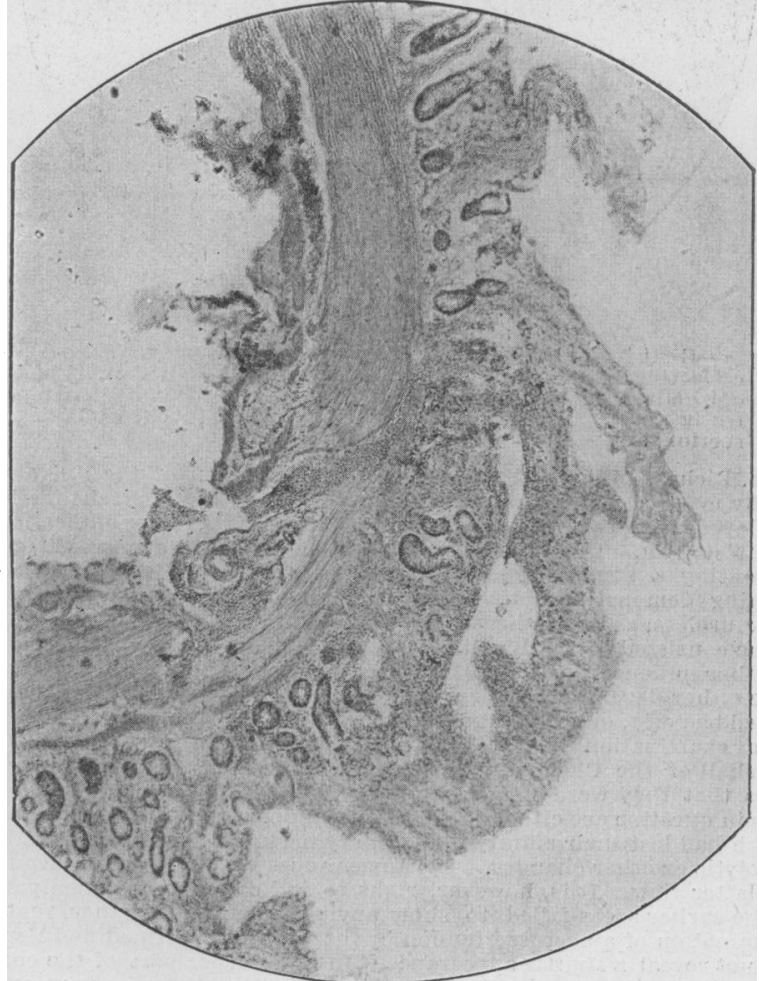

ig. 2.-Gout in fowl. Small intestine. The mucous membrane show3, $a$, diminution in the glandular elements; $b$, increased amount of lymphoid tissue ; and $c$, catarrhal exudation on the free surface.
5. Marked congestion of the liver, lungs, and kidney. Microscopic examination of the liver showed some atrophy of the liver cells. The congestion of liver is indicated in Fig, $I$.

6. Areas of necrosis in the kidneys. Scattered throughout the organ there were numerous areas of necrosis (see Fig. 5). which varied in size from the head of a pin up to a small bean. these presented a white amorphous appearance quite distinct from the rest of the organ. The

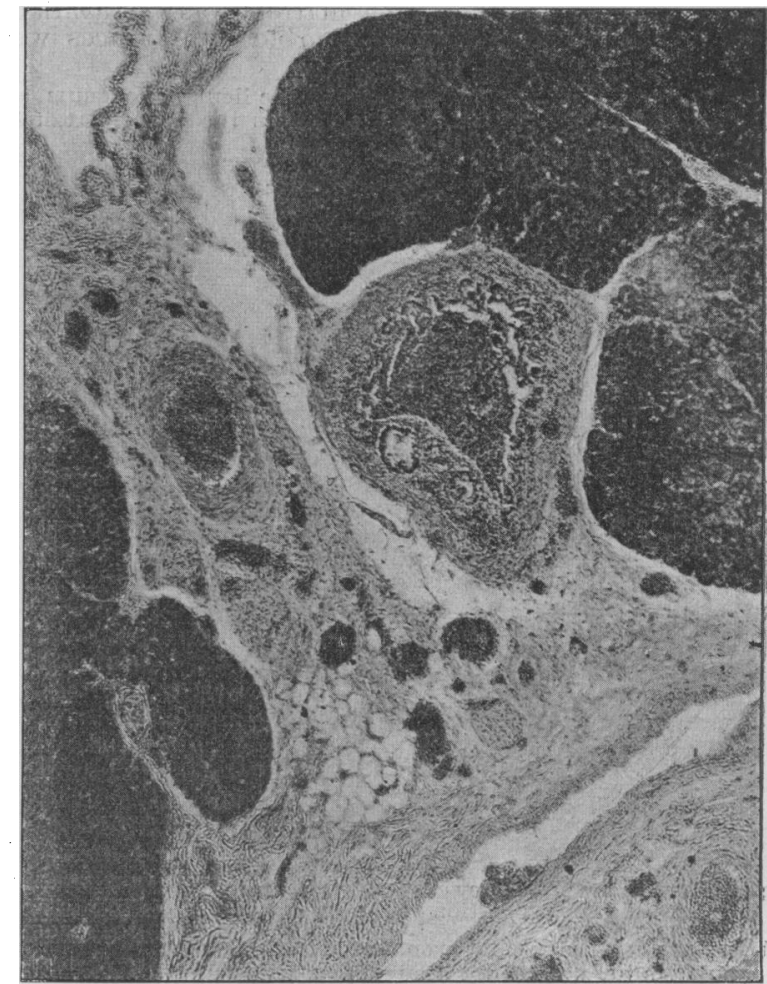

Fig. 3.-Gout in fowl. Pancreas. Transverse section at the hilum: The lumen of the duct is tilled by catarrhal cells derived from the anflamed mucous membrane; the vessels in the wall of the duct

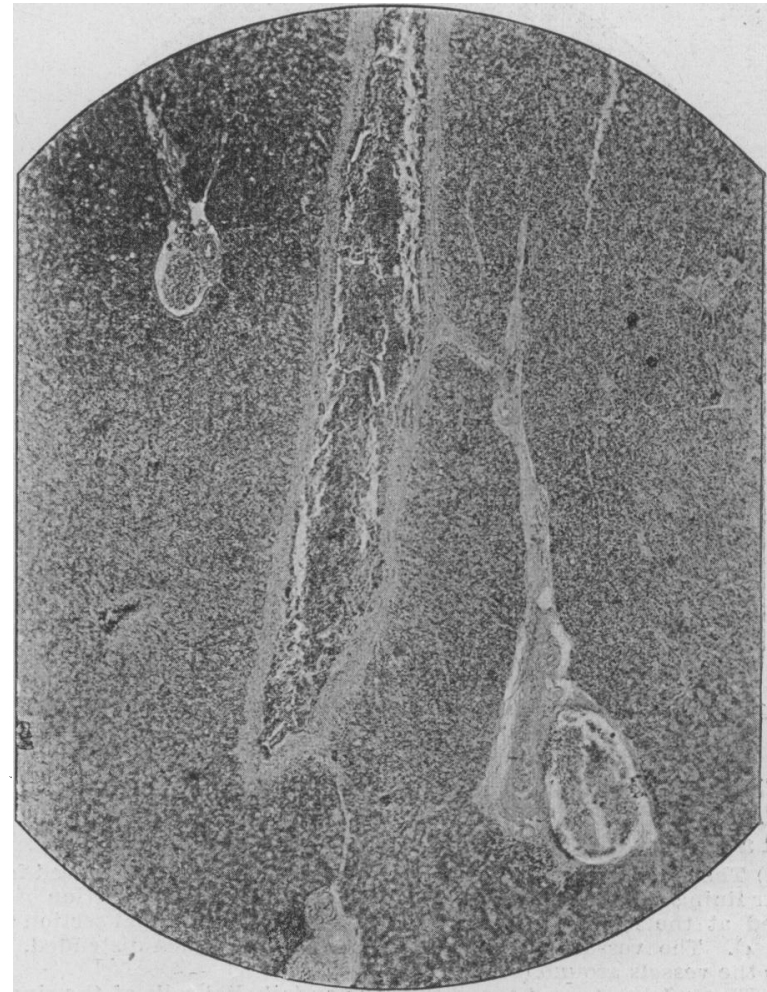

Fig. 4.-Gout in fowl. Pancreas. Longitudinal section. (Note the blocking of the lumen of the duct by catarrhal products.) 
cortex was markedly congested (see Fig. 5). The histological characters of the kidney are described later.

A microscopic examination was made of the foregoing and other tissues-namely, intestinal tract, pancreas, lymphatic glands, spleen, sciatic arteries and nerves, skin, comb, and testicle, the tissues of a healthy fowl of the same age being used: for comparisons. The bone marrow was not available for examination. The following morbid appearances were found :

(a) Catarrh of the intestine, especially in the ileum, duodenum, and large intestine. This was of a patchy nature in the upper part of the

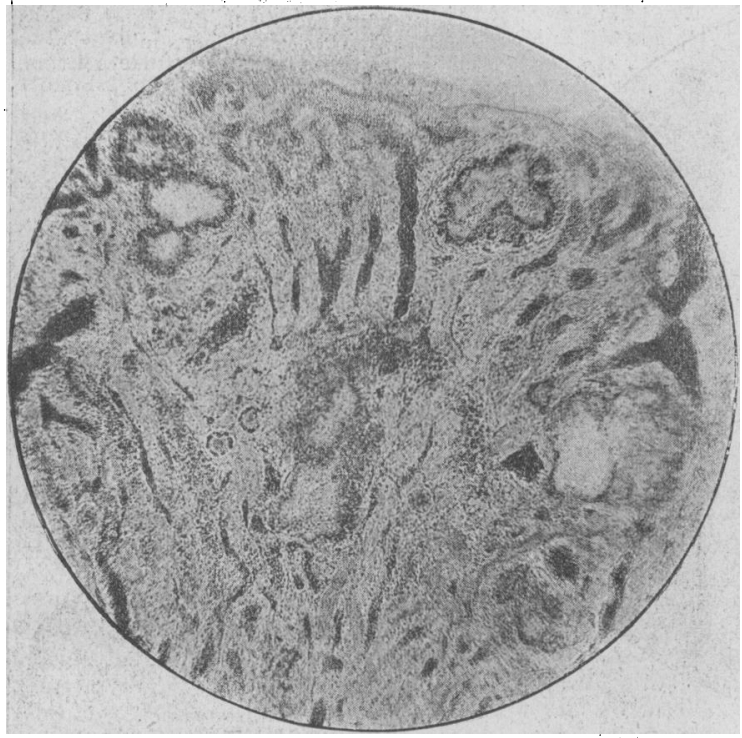

Fig. 5.-Gout in fowl. Kidney cortex. (Observe the areas of necrosis (see text) and the congestion of the organ.)

tract, but was continuous and pronounced in the lower end of the ileum. The capillaries were markedly dilated, apparently due to a condition of venous stasis. There was a great excess of lymphoid tissue in the ileum.



Fig. 6.-Gout in fowl. Kidney at junction of cortex and medulla. The dark bands are dilated collecting tubules full of granular leucocytes. The blood vessels have been blocked out of the section.

Fig. 2 shows a loss of glandular structures with a separation of the glandular elements by excess of interglandular tissue, and a catarrhal exudation lying on the free surface.

(b) The pancreatic duct was filled by catarrhal products derived from their lining membrane. This is well seen in a transverse section of the gland at the hilum (see Fig. 3) and also in a longitudinal section (see Fig. 4). The vessels in the wall of the duct were much distended, and also the vessels around (see Fig. 3 ).

(c) The spleen showed congestion, the endothelial"cells of the sinuses were proliferated, and there was a marked increase in the number of granular leucocytes in the capillaries and sinuses as compared with the control sections.

These features are the characteristic reaction of this organ to invasion by bacteria or their products.

(d) A lymphatic gland showed a minute crystalline deposit of urate of soda surrounded by a few small round and epithelial cells.

This point is of interest in connexion with views previously held as to the involvement of lymphatic glands in cases of gout. In his well-known work Duckworth states that " the lymphatic system has been held to be free from any changes in gout. The glandular portion cannot be said to be involved, but there is clinical evidence of subacute gouty inflammation of lymph spaces in certain organs due to uratic deposit and influence. Dr. Buzzard has called attention to this.

(e) The kidneys. Fig. 5 shows the appearance of the cortex of the kidneys under a magnification of 20 diameters. Note four necrotic areas in the field. These present a whitish colour, and are surrounded by a dark ring which consists of small round cells of an inflammatory nature. The small dark points visible in the centre of the areas are granular leucocytes which have invaded the necrosed areas. In fowls granular leucocytes which have invaded the necrosed areas. In fowls those leucocytes are large and possess very coarse "crystalline"
granules. The dark bands in the figure are congested blood vessels. Under a magnification of 600 diameters with ordinary stains, for ex ample, haematoxylin, eosin, and methyl blue, these necrotic areas presented a structureless homogeneous appearance. An examination of smaller and earlier nodules revealed at parts a eonsiderable amount of crýstalline deposit of sodium urate surrounded by inflammatory tissue. It was not possible for me to express a definite opinion as to whether their original starting point was in the tubules or in the intertubular tissue. The arrangement of these smaller areas alongside the interlobular arteries suggested an infection by the blood stream.

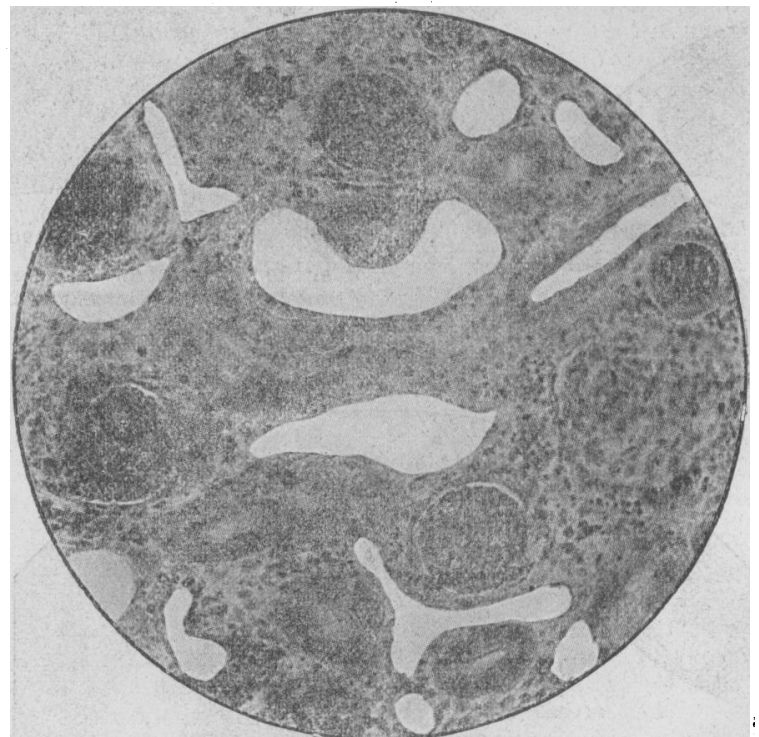

Fig. 7-Gout in fowl. Kidney at deep part of the cortex. (Note (r) the collecting tubules full of granular leucocytes, with atrophy of the epithelial lining: (2) several convoluted tubules which appear per-
fectly normal. The blood vessels have been blocked out of the section, and appears white. See also Fig. 6.)

Mr. Richard Muir, of the Pathological Department of the University, kindly made a bacteriological examination of the sections, the methods employed being ( $\mathrm{r}$ ) the ordinary basic stains, and (2) a modification of Benda's stain. The former gave negative results, the necrosed areas presenting a structureless hyaline appearance; the latter method of staining demonstrated that the tissue in the necrotic areas was not tissue consisted of rod-like bodies of the size of large bacilli massed together in dense clusters; the appearance suggested that these rods were either degenerated cell products of an unusual character, degenerated bacteria, or crystalline in nature.

An examination with a polariscope, kindly made for me by Dr. Marshall of the Chemistry Department of the University, clearly indicates that they were not crystalline. We therefore conclude that the rods in question are either the products of degenerated cells or bacteria which had lost their affinity for ordinary bacterial stains, owing to bacteriolytic or other changes. The appearances generally are in favour of the latter view. It is, however, right to indicate that an examination of the earlier areas failed to show any bacteria; and, further, that an examination of a necrosed tendon by the same method (modified Benda) did not reveal a similar appearance. In the deeper part of the cortex and in the medulla a very striking pathological condition was present in the collecting tubules. These were everywhere markedly dilated and 
Fig. 6, in which the dark bands are collecting tubules. For the sake of clearness the vessels have been blocked out of this and the subsequent figure. These granular cells can be seen emigrating from surrounding capillaries through the walls of the sollecting tubules. The renal blood vessels contained a marked excess of these blood constituents. This condition of the collecting tubules is also indicated in Fig. 7, which shows in addition the following features

(a) The presence of granular leucocytes lying free in the intertubular

(b) An atrophy of the epithelial Jining of the convoluted tubules, doubtless due to pressure of the leucocyte exudation.

(c) Several convoluted tubules which appear perfectly normal.

A transverse section of the ureter in the same section from which

Fig. 6 was taken shows that the leucocytes had undergone marked degeneration in the course of their downward journey, very few leucocytes being present as such in the ureter.

The gouty deposit in the tissues, the changes in the synovia, the widespread thromboses, and the areas of necroses in the kidney clearly indicate the nature of the disease. It is a typical case of gout. It is furthermore a case of acute gout which had proceeded to its natural termination. The fact that early indications of pericarditis were present might suggest the opinion that the animal. undoubtedly the subject of gout, succumbed to some terminal infection not immediately related to the gouty disorder. The clinical history and pathological appearances alike fail to substantiate this view. In this connexion reference may be made to the :

1. Absence of any alteration in the joint structures other than in the synovia, the nutritive fluid of the joint. There was no gouty arthritis nor other indication of chronic disease.

2. The absence of any cirrhotic changes in the kidney and fibrous overgrowth in relation to the deposits in the extraarticular tissues such as we would expect in chronic disease.

3. The blood vessels of the animal were singularly healthy, which in all probability would not have been in a case of chronic disease that had succumbed to what is commonly known as a terminal infection.

These negative facts fail to substantiate this hypothetical view as to the existence in this fowl of two or more diseases not etiologically related, and this failure is emphasized by reference to some of the other unusual features of this case, more especially to the condition of the pancreas and collecting tubules of the kidney, both of which present pronounced morbid appearances of a character extremely rare in the history of simple terminal infection. We are therefore driven to the conclusion that we have to deal with a case of pure gout in which thc disease had run a natural course, in which an examination of the tissues shows that the postmortem appearances generally are characteristic of an infection by bacteria or their products.

Particular interest centres in the condition of the intestinal tract, pancreas, and kidneys. The condition of the pancreatic duct is a very striking one ; it appeared to be an extension from the duodenum. A reference to the figures shows that the lesion would be in its results analogous to the effect of a ligature of the duct. The interesting question is raised as to the existence of any relationship between these appearances and the occurrence of gouty glycosuria in the human subject. The changes in the tubules of the kidney are remarkable. I am not aware that these appearances have been previously described. The alterations in the leucocytes have a special interest in connexion with the changes in the blood in acute polyarticular gout recently described by the author $^{2}$ and confirmed by Bain. ${ }^{3}$

The main facts learned from this investigation emphasize the necessity of further examination into the comparative pathology of the disease. It is specially important to obtain material sufficiently fresh to allow of an adequate bacteriological investigation. A question that at once presents itself from the foregoing record is, How would the acceptance of the theory of a bacterial origin of the disease harmonize with the known clinical facts of the disorder? The consideration of this point is not relevant to this communication. It may, however, be stated that in my opinion the clinical features of gout-regular or irregular, acute or chronic-are more adequately explained by the light of our present knowledge of infections, relapses, and immunity than by any other theory. The distinctive feature of the infection in gout is that the toxin or toxins have the special property of disturbing nitrogenous metabolism in a manner favourable to the deposit of uric acid in certain tissues.

In conclusion, I would only add that the addition of a bacterial factor to our present conception of the disease would in no way minimize the importance of attention to dietetics in its treatment. It would correct the teaching of some writers whose standard of dietary has special reference to nuclein-holding foods, and would focus attention on the by-products of deranged digestion of carbohydrates and proteids arising from abnormal bacterial activity in the digestive tract and other tissues.

1 Encyclopaedia Medica, vol. iv, article Gout. 2 BRITISH MEDICAL JouRNAL, January 6th, I900. 3 Ibid., January 31st, 1903.

\section{GLANDULAR FEVER.}

BY JOHN W. BYERS, M.A., M.D.

Professor of Midwifery and of Diseases of Women and Children, Queen's College. Belfast ; Yhysician for Diseases of Women, Royal Victoria

Hospital. Belfast; Physician, Belfast Maternity Hospital ; and Consulting Physician, Belfast Hospital for sick Children.

Since the middle of November an epidemic has been prevailing, chiefly among children, in Belfast and in parts of the North of Ireland of a kind which I have not sten before, either in private practice or during the years that I was physician to the Belfast Hospital for sick Children, and which seems to me to correspond to the condition described in 1889 by Pieiffer, and termed often by German writers Pfeiffer's Drïsenfieber. $O$. Heubner, Dawson Williams, Comby, Donkin, and J. P. West have also contributed largely to the literature of glandular fever. My knowledge is based on 33 cases, some of which occurred in two public institutions, while the others were seen in consultation.

Age.- The youngest ehild was I 3 montha, the oldest case was a female servant, aged 25 , in a family where the children were attacked ; but, with the exception of two cases (the one mentioned and another aged 21), all were in children or young people under 16 years.

Onset.--As a rule this was sudden.

Period of Incubation.- This was from five to seven days.

Symptoms.-In some of the cases the children went to bed in the evening complaining of slight sore throat. The next morning there was sharp fever $\left(102^{\circ}\right.$ to $104^{\circ} \mathrm{F}$.), pain on one side of the neck, which was tender on pressure and held stiffly; this was succeeded in another twelve hours by a distinct swelling of the glands under and in front of the sterno-mastoid. In the majority of the cases a similar enlargement appeared involving the deep glands of the corresponding and opposite sides of the neck. In the cases seen by me the right side was as a rule first affected. In another group of those attacked the children complained of nothing until the glands of the neck seemed suddenly to enlarge, and such cases when seen at first looked somewhat like aberrant forms of "mumps." In the severer cases headache was an initial symptom, and in a few sickness and vomiting were present and some abdominal pain, but I failed to detect any olijective signs of involvement of the mesenteric glands. In none of the cases was the throat in any way affected beyond some slight congestion, and in the majority it was normal and showed no alteration to explain the slight dysphagia. The glands affected were as a rule the deep set under and in front of the sterno-mastoid, but in others those in the posterior triangles of the neck were involved; no suppuration occurred in any of the casts. and the swelling of the glands subsided in most of the cases within a week from the onset of the attack, but in one case the enlargement remained for a fortnight, the fever going up and down in an erratic manner most of that time. The majority of the children were convalescent in a week, but in some of the severe cases those attacked remained poorly and looked anaemic for three to four weeks the glands continuing enlarged. ' When the disease entered a house nearly all the children were attacked, and in one place two of the female servants, both adults. No rash appeared in any of the cases, nor was there any kidney or other complication, and none suffered from depression except the very severe cases. There were no deaths.

Diagnosis. - When the first case appeared in a house, the adenitis naturally suggested some primary throat mischief, but nothing sufficient to cause the glandular swellings could be discovered on an examination of the throat, either on the tonsils or pharynx, and the presence of other cases in the same house showed that one had to deal with an epidemic fever.

Etiology.-In the British Medical Journal of December th, 1903, p. 1492, there is described an "epidemic sore throat and suppurative mammitis in cows," but in none of the cows seen by me were theie any such conditions observed in the throat, and the cases were in groups, widely separated, and with no common milk supply. The mild type of cases, when 